\title{
How the carrier momentum influences the polarization properties of a vertical-cavity semiconductor laser
}

\author{
R. F. M. Hendriks, M. P. van Exter, and J. P. Woerdman \\ Huygens Laboratory, Leiden University, P.O. Box 9504, 2300 RA Leiden, The Netherlands
}

(Received 18 June 1998)

\begin{abstract}
By taking carrier momentum into account, we present an extension of the commonly used model that describes the polarization properties of vertical-cavity surface-emitting lasers (VCSEL's). We study how this modifies the polarization dependence of the gain saturation, and introduce the role of the crystalline symmetry of the semiconductor material. On a macroscopic level we show that a limited number of parameters is sufficient to describe fully the polarization properties of the VCSEL. On a microscopic level we show how the magnitudes of these parameters are determined by the dependence of the interband-transition dipole matrix element on the relative orientation of the carrier momentum and optical polarization. It turns out to be essential that the component of the carrier momentum in the plane of the quantum well is non-negligible. Furthermore, the relaxation rates of the carrier momentum and carrier spin are crucial in determining the magnitude of the polarization effects. Inclusion of the carrier momentum changes the interpretation of experimental results on the polarization of VCSEL's, and makes it possible to understand experimental results that were until now unexplained. [S1050-2947(99)02201-5]
\end{abstract}

PACS number(s): 42.55.Px

\section{INTRODUCTION}

Vertical-cavity surface-emitting lasers (VCSEL's) are a very interesting breed of semiconductor lasers, both fundamentally and from an applications point of view. This is particularly true for the polarization properties which are directly linked with the inherent transverse geometry. In a VCSEL the quantum wells (QW's) are oriented perpendicular to the propagation direction of the light, so the field is always polarized in the plane of the QW. This situation is totally different from that in edge-emitting semiconductor lasers, where there is generally a large difference in gain between TE- and TM-polarized light, i.e., light that is linearly polarized in the plane of the $\mathrm{QW}$ or perpendicular to it, and where the device geometry determines the optical polarization. In most practical VCSEL's the optical polarization is determined by the birefringence, which originates from the electro-optic effect and from stress and strain induced by the electrical contacting of the devices [1,2]. This birefringence in VCSEL's creates a frequency difference between the two orthogonally polarized modes. Apart from birefringence there is also some dichroism present, which causes a gain or loss difference between two orthogonally polarized modes [3]. Together, birefringence and dichroism can account for most of the polarization properties of VCSEL's [3].

Birefringence and dichroism are linear anisotropies, i.e., they do not depend on saturation of the inversion in the VCSEL gain medium. There are, however, also nonlinear (saturation induced) anisotropies. These nonlinear anisotropies exist due to the fact that the saturation intensity is different for linearly polarized light and circularly polarized light. In QWs the influence of these saturation-induced anisotropies has been studied theoretically [4-6] and experimentally $[7,8]$. The framework for these studies has been the model developed by San Miguel, Feng, and Moloney (SFM) in 1994. This SFM model is basically a gas laser model; the interband transition between valence and conduction bands is replaced by a transition between discrete energy levels, which are the Zeeman magnetic sublevels. The quantum numbers of these levels are chosen to agree with the QW band structure. The total carrier density is divided into two carrier reservoirs, that couple with left- or right-handed circular polarizations only. In the SFM model the relaxation rate of population differences between these reservoirs is included by a phenomenological spin-flip parameter $\Gamma_{s}$. This is the crucial parameter in the model; it determines how the saturation depends on the polarization. Recent experimental work has shown that $\Gamma_{s} \approx 300[7,8]$, which means that the difference between the saturation intensity for circularly polarized light and linearly polarized light is roughly $0.3 \%$.

In the SFM model the QW is treated as if it is an "ideal" $\mathrm{QW}$, which is infinitely thin, so that the carrier momentum is completely dominated by the perpendicular, confinementinduced, component. Band-structure aspects of the QW gain medium, that will appear if the in-plane component of the carrier momentum is nonzero, are not taken into account beyond using the "proper" quantum numbers for the discrete levels. The assumption that the in-plane component of the carrier momentum is negligible, is not correct in practice. In fact, the in-plane and perpendicular components of the carrier momentum in the plane and perpendicular to it are of the same order of magnitude. We will discuss two effects that originate from the in-plane component of the carrier momentum: (i) momentum alignment hole burning, and (ii) the influence of the cubic symmetry of the crystalline structure.

Effect (i) has been studied previously in pump-probe experiments on bulk GaAs [9]. It was observed that a linearly polarized light pulse preferentially couples with carriers that have their in-plane momentum aligned perpendicular to the optical polarization. A linearly polarized pulse thus creates a nonequilibrium carrier momentum distribution, i.e., it burns a "hole" in the momentum distribution of the carriers, which results in a (pump-induced) birefringence and dichro- 
ism. This effect is not to be confused with the more familiar spectral hole burning, where one deals with a nonequilibrium energy distribution of the carriers. The decay rate of the "hole" in the momentum distribution is determined by the momentum relaxation rate. The influence of the momentum alignment hole burning on the polarization state of active devices, like VCSEL's, has not been studied previously.

Effect (ii) deals with the relative orientation of the optical polarization and the crystalline axes. It is important to note that a system with a cubic symmetry (like the III-V semiconductor compounds) is fully isotropic in a linear-optical sense. However, the crystalline orientation will show up in the nonlinear anisotropies, as the saturation intensity can depend on the orientation of the polarization with respect to the crystalline axes [10-12].

Although it is generally recognized that the SFM model is incomplete in neglecting band-structure aspects, most experimental results have nevertheless been rather successfully interpreted within the model. The only experimental result reported so far that is inconsistent with this model, in a qualitative sense, was performed on optically pumped VCSEL's. It was found that the polarization orientation of linearly polarized pump light influences the polarization orientation of the VCSEL output [8]. In this paper it will be shown how including band-structure aspects changes the interpretation of the experimental results with respect to the SFM model, and how the observed dependence on the pump polarization can be explained. Furthermore we will explain why, in spite of neglecting band-structure aspects, the SFM model is generally so successful.

The extension of the SFM model as presented in this paper applies to all QW VCSEL's, electrically as well as optically pumped. The reason that we refer mostly to experiments performed on optically pumped VCSEL's is that in these experiments the influence of the carrier momentum is best visible. In electrically pumped devices the effects associated with the carrier momentum are less suitable as a demonstration, mainly because of the large uncertainty in the parameters involved.

One goal of this paper is to keep the model as simple as possible by extending the SFM model with one aspect only, namely, the in-plane carrier momentum. This is done by introducing additional carrier reservoirs, apart from those in the SFM model. These reservoirs each couple with orthogonal linear polarizations. A set of rate equations describes the population transfer between the reservoirs, and the coupling with the optical field. We do not include many-body aspects like band-gap renormalization and carrier screening. Furthermore, as we are interested in polarization properties we neglect band-structure effects associated with the isotropic carrier distribution, i.e., spectral hole burning, carrier heating, and nonlinear gain. So we assume that the carrier distribution over the energy levels is in equilibrium. Although these effects might be important, we do not take them into account because they obscure the polarization physics associated with our extension of the SFM model.

In Sec. II, we will present a short macroscopic discussion based on crystalline symmetry. Section III contains a microscopic discussion, based on the semiconductor Bloch equations. In Secs. IV and V the microscopic and macroscopic approaches are combined, and the consequences of the ex- tension in a rate-equation approach are discussed. Conclusions are presented in Sec. VI.

\section{MACROSCOPIC CONSIDERATIONS}

This section deals with macroscopic considerations on the polarization properties of the QW gain medium in a VCSEL based on third-order (Lamb-type) laser theory [13]. Due to the high symmetry of the GaAs crystalline lattice, a limited number of independent parameters is sufficient to fully characterize the optical polarization properties of the VCSEL device. In this section we will identify the role of these parameters. We consider the population inversion to be eliminated adiabatically, i.e., the laser is treated as if it was a class-A laser. One might think that this is not valid in VCSEL's, since the decay rate of the inversion is much smaller than the decay rate of the field in the cavity. It has been shown, however, that if one is only interested in the polarization of the VCSEL, adiabatic elimination is allowed [6], since the relaxation rates of the magnetic sublevels associated with polarization properties are extremely fast.

The linear anisotropies are fully described by the first order susceptibility tensor. In the most general case this $2 \times 2$ tensor has four complex elements, and one needs eight real parameters to fully characterize the linear anisotropies. A cubic crystal, however, is fully isotropic for any linearoptical effect (i.e., the unsaturated gain or loss is independent of the optical polarization). The first-order susceptibility tensor is then diagonal, with identical elements [11]. The presence of a QW will break the symmetry between the directions perpendicular and parallel to the QW. In the plane of the QW the symmetry is maintained. The in-plane symmetry can be broken, for instance, by the pump process [8], by uniaxial strain in the plane of the QW [14], by a perpendicular electrical field [15] or by growth or misoriented substrates [16]. In this case of broken symmetry the time evolution of the complex amplitude of the optical field does in principle depend on the polarization state of the light.

For the nonlinear anisotropies there are, in a system with cubic symmetry, generally three different eigenpolarizations, i.e., polarization states that do not change when propagating through the system [11]. For an optical field propagating along one of the crystalline axis (which we take to be the [100] axis) these eigenpolarizations are circular polarization, linear polarization along [001], and linear polarization along [011], which is at $45^{\circ}$ to the [001] direction. The nonlinear anisotropies are characterized by the differences in nonlinear refractive index $n_{2}$, and the nonlinear absorption coefficient $\beta$, for these eigenpolarizations. In the literature the difference between circular and linear polarization is standardly defined by the parameters $\delta_{r, i}[10]$ :

$$
\begin{aligned}
& \delta_{r}=\frac{n_{2}^{(0)}-n_{2}^{(\mathrm{circ})}}{n_{2}^{(0)}}, \\
& \delta_{i}=\frac{\beta^{(0)}-\beta^{(\mathrm{circ})}}{\beta^{(0)}},
\end{aligned}
$$

and the orientational anisotropy is described by $\sigma_{r, i}$ as 


$$
\begin{gathered}
\frac{1}{2} \sigma_{r}=\frac{n_{2}^{(0)}-n_{2}^{(45)}}{n_{2}^{(0)}}, \\
\frac{1}{2} \sigma_{i}=\frac{\beta^{(0)}-\beta^{(45)}}{\beta^{(0)}} .
\end{gathered}
$$

Here the superscript (0) denotes light linearly polarized along [001], (45) denotes light polarized along [011], and (circ) denotes circularly polarized light. The equation describing the optical field evolution for an eigenpolarization is then

$$
\frac{d E^{p}}{d z}=+i \frac{\omega}{c}\left(n+n_{2}^{p} I\right) E-\frac{1}{2}\left(\alpha_{g}+\beta^{p} I\right) E
$$

where $p$ denotes the eigenpolarization considered, $I$ the optical intensity, $n$ the linear refractive index and $\alpha_{g}$ the lossgain coefficient.

If the polarization is not an eigenpolarization, the parameters $\delta_{r, i}$ and $\sigma_{r, i}$ determine the evolution of the polarization state. Nonzero values for $\delta_{i}$ and $\sigma_{i}$ correspond to a nonlinear dichroism: the polarization will evolve toward the eigenpolarization that has the least saturation. If $\delta_{i}>0$, an arbitrary elliptical polarization becomes circularly polarized in the course of time; if $\delta_{i}<0$ it will become linearly polarized. In the same way $\sigma_{i}$ corresponds, depending on the sign, to a preference for either [001] or [011] linear polarization. The parameters $\delta_{r}$ and $\sigma_{r}$ correspond to a nonlinear birefringence, which causes a noneigenpolarization state to repetitively change its ellipticity and/or orientation.

It is interesting to discuss the values of the $\delta_{r, i}$ and $\sigma_{r, i}$ parameters for the SFM model. In the SFM model, $\delta_{r, i}$ is fully determined by the spin-flip parameter $\Gamma_{s}, \delta_{r}=\delta_{i}=$ $-1 / \Gamma_{s}[4,6]$, where $\Gamma_{s}$ is estimated to be roughly $300[8,7]$. The fact that $\delta_{r}$ and $\delta_{i}$ have equal values indicates that the relative dependence of the saturation intensity on the polarization is identical for the nonlinear dichroism and the nonlinear birefringence. The large value of $\Gamma_{s}$ causes the saturation intensity to depend very little on the polarization. In the SFM model the QW VCSEL is treated as if the underlying crystalline symmetry was fully isotropic instead of cubic. Therefore, the saturation does not depend on the orientation of the polarization, and $\sigma_{r, i}=0$. In Sec. III we will show, from a microscopic analysis, that the symmetry parameters $\delta_{r, i}$ and $\sigma_{r, i}$ depend on the details of the semiconductor band structure, and that, if this band structure is taken into account, the cubic symmetry of the crystal is reflected in the polarization dependence of the saturation.

\section{MICROSCOPIC CONSIDERATIONS}

In this section we present a microscopic analysis of the band-structure aspects of the QW gain medium. The crucial point in our analysis is that the carrier momentum has a component in the plane of the QW. In the existing model for VCSEL polarization this fact is not taken into account [4]. The interband transition dipole element depends on the magnitude and the orientation of the carrier momentum. We will first consider the transition for each carrier momentum state separately; in the end we will integrate over all momentum states in order to describe the effect of the QW as a whole.
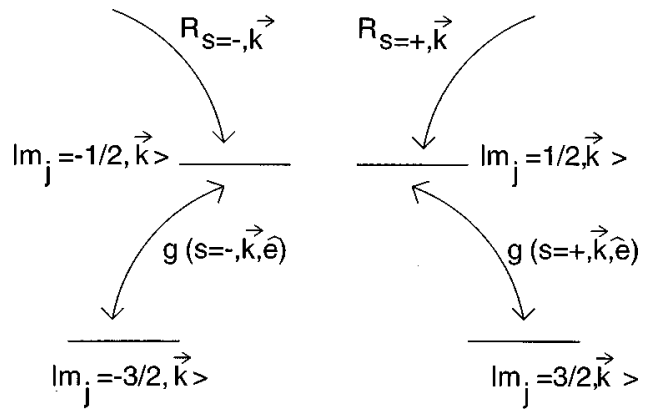

FIG. 1. Level scheme for carrier with momentum $\hat{k}$.

Figure 1 shows the level scheme corresponding to one single momentum state. Here the $m_{j}= \pm \frac{1}{2}$ levels denote the conduction band states, while the $m_{j}= \pm \frac{3}{2}$ levels denote the heavy-hole $(\mathrm{HH})$ valence band. The carrier momentum is denoted by the vector $\vec{k}$. The transition strength, which is defined as the square of the interband transition dipole element, is denoted by $g(s, \vec{k}, \hat{e})=|\vec{\mu}(s, \vec{k}) \cdot \hat{e}|^{2} /\left|\mu_{0}\right|^{2}$. The spin label $s$ denotes whether the transition is between the levels with $m_{j}=+\frac{3}{2}$ and $m_{j}=+\frac{1}{2}(s=+)$ or $m_{j}=-\frac{3}{2}$ and $m_{j}=$ $-\frac{1}{2}(s=-)$. The optical polarization is denoted by $\hat{e}$. The dipole elements have been normalized with respect to the transition between the bottom of the conduction band and the top of the valence band for right-hand circularly polarized light. The pump rates to the upper levels are denoted as $R_{s, \vec{k}}$. Note that the electron and hole states that are coupled by the optical transition have approximately the same momentum, as the photon momentum is relatively small in this process. We only consider transitions between the lowest-energy bound states in the QW.

By taking the valence band to be the $\mathrm{HH}$ band we have neglected band mixing. Generally in a QW the valence band consists of a mixture of the $\mathrm{HH}$ and light-hole (LH) band. The amount of LH that is mixed into the valence band depends on the energy splitting between the $\mathrm{LH}$ and $\mathrm{HH}$ bands, and also on the magnitude of the in-plane component of $\vec{k}$. Whether neglecting band mixing is a valid approximation depends on device architecture, i.e., the thickness of the QW, the composition of QW and cladding layers, and the biaxial strain in the QW. At the end of this paper we will discuss how band mixing can be included into our model, and what consequences this will have.

The rate equations for the transition in Fig. 1 can be written as

$$
\begin{aligned}
\dot{E}= & -\kappa E+\kappa(1-i \alpha)\left(\sum_{s, \vec{k}} g(s, \vec{k}, \hat{e}) N_{s, \vec{k}}\right) E, \\
\dot{N}_{s, \vec{k}}= & -\gamma\left(N_{s, \vec{k}}-R_{s, \vec{k}}\right)-2 \gamma g(s, \vec{k}, \hat{e})|E|^{2} N_{s, \vec{k}} \\
& -\gamma_{j} \sum_{s^{\prime}}\left(N_{s, \vec{k}}-N_{s^{\prime}, \vec{k}}\right)-\gamma_{k}\left(N_{s, \vec{k}}-\left\langle N_{s, \vec{k}}\right\rangle\right) .
\end{aligned}
$$

Here $E$ denotes the slowly varying amplitude of the optical field, while $\hat{e}$ denotes the optical polarization, so that the time-dependent optical field vector is $\vec{\varepsilon}(t)=e^{-i \omega t} E(t) \hat{e}(t)$. $N_{s, \vec{k}}$ is the population inversion. In Eq. (6), $\kappa$ is the cavity loss rate and $\alpha$ is the linewidth enhancement factor. In Eq. 


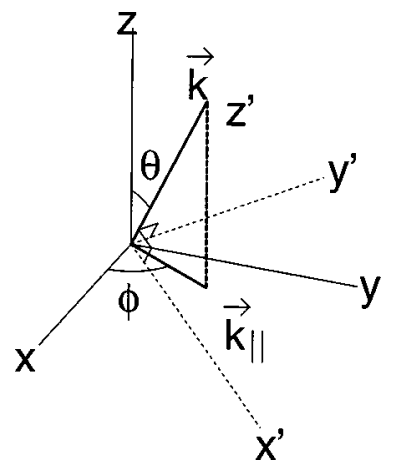

FIG. 2. Orientation of carrier reference frame $x^{\prime} y^{\prime} z^{\prime}$ with respect to the QW reference frame $x y z$. The carrier momentum $\vec{k}$ is oriented along $z^{\prime} \cdot \vec{k}_{\|}$is the component of the carrier momentum in the plane of the QW ( $x y$ plane).

(7) the parameter $\gamma$ determines the population inversion decay rate and $\gamma_{j}$ denotes the relaxation rate between different spin levels. Furthermore $\gamma_{k}$ describes the relaxation rate toward the equilibrium carrier momentum distribution, which is denoted by $\left\langle N_{s, \vec{k}^{\prime}}\right\rangle$ and depends on the pump rate and the optical intensity. The units of the population inversion and the pump rate have been chosen so as to normalize the sum: at the lasing threshold $\sum_{s, \vec{k}} N_{s, \vec{k}} \approx \sum_{s, \vec{k}} R_{s, \vec{k}} \approx 2$. The factor 2 in this normalization arises from the fact that we require that in Eq. (6) the overall gain is positive for both circularly polarized components of the optical field.

In this model there is only room for one spin-flip parameter, describing the population transfer between the carrier reservoirs for left- and right-handed circular polarizations, i.e., between the left- and right-hand side of the level scheme in Fig. 1. Because the population transfer is included phenomenologically, it is not relevant whether it occurs via the holes or via the electrons. As stated above, the new element in the rate equations is the inclusion of $\vec{k}$. The parameter $\gamma_{k}$ models, in a phenomenological way, the relaxation processes associated with the carrier momentum. If this relaxation is extremely large the population distribution over the momentum states is always in equilibrium, so $N_{s, \vec{k}}=\left\langle N_{s, \vec{k}^{\prime}}\right\rangle$. In this situation we have Eqs. (6) and (7).

The important parameter in the rate equations is the normalized transition strength $g(s, \vec{k}, \hat{e})$, which depends on carrier spin, carrier momentum, and optical polarization. Of special importance is the relative orientation of carrier momentum and the optical polarization. In fact, it is convenient first to express the transition strength in a reference frame in which the carrier momentum is along the $z$ axis. In Fig. 2 the carrier momentum reference frame is denoted by $x^{\prime} y^{\prime} z^{\prime}$. The QW reference frame, or lab frame, is denoted by $x y z$. The angles $\theta$ and $\phi$ define the orientation of the carrier momentum. $\theta$ is the angle between the $\hat{z}$ direction and the carrier momentum, and $\phi$ defines the orientation of the component of $\vec{k}$ that is in the plane of the quantum well, such that $k_{\|} e^{i \phi}=k_{x}+i k_{y}$. In a $\mathrm{QW}$ the component of the carrier momentum perpendicular to the $\mathrm{QW}$ is fixed due to the potential well, so summations over $\vec{k}$ are effectively summations over $\vec{k}_{\|}$.
Expressed in the $x^{\prime} y^{\prime} z^{\prime}$ reference frame in Fig. 2, the transition strength for a transition between $\mathrm{HH}$ and conduction band is given by [17]

$$
g\left(s= \pm, \vec{k}_{\|}, \hat{e}\right)=\frac{1}{2}\left|e_{x}^{\prime} \pm i e_{y}^{\prime}\right|^{2}
$$

where $e_{x, y}^{\prime}$ denotes the optical polarization components along the $x^{\prime}$ and $y^{\prime}$ axes, i.e., the optical polarization as experienced by the carriers with momentum $\vec{k}$.

Now a simple coordinate transformation of the $x^{\prime} y^{\prime} z^{\prime}$ frame into the $x y z$ reference frame (the $\mathrm{QW}$ reference frame) yields the coupling strength as a function of $e_{x}, e_{y}$, and $e_{z}$, which can be expressed in terms of the Stokes parameters as

$$
\begin{aligned}
g(s= \pm)= & \frac{1}{2}\left[\frac{1}{2}\left(1+\cos ^{2} \theta\right)-\frac{1}{2} \sin ^{2} \theta\left(S_{1} \cos 2 \phi\right.\right. \\
& \left.\left.+S_{2} \sin 2 \phi\right) \pm S_{3} \cos \theta\right] .
\end{aligned}
$$

The sign of the " $S_{3}$," term depends on the transition considered $(+$ for $s=+,-$ for $s=-) . S_{1,2,3}$ are the normalized Stokes parameters defined in the $Q W$ reference frame, i.e., the $x y z$ frame in Fig. 2, as

$$
\begin{gathered}
S_{1}=\left|e_{x}\right|^{2}-\left|e_{y}\right|^{2}, \\
S_{2}=e_{x}^{*} e_{y}+e_{y}^{*} e_{x}, \\
S_{3}=i e_{x}^{*} e_{y}-i e_{y}^{*} e_{x} .
\end{gathered}
$$

Here $e_{x}\left(e_{y}\right)$ is the component of the polarization vector along the $x(y)$ axis, and $\left|e_{x}\right|^{2}+\left|e_{y}\right|^{2}=1$.

We thus find that, for $k_{\|} \neq 0$, the coupling strength depends on the orientation of the optical polarization with respect to the orientation of the carrier momentum. The optical field couples optimally with carriers that have their momentum aligned orthogonal to the polarization direction: if the carrier momentum $\vec{k}_{\|}$is along the $\hat{x}$ axis the coupling strength is maximum for light linearly polarized along the $y$ axis (in this case $\phi$ is zero, and $S_{1}=-1, S_{2}=0$, and $S_{3}$ $=0$ ) reduce to the original equations of the SFM model as in Refs. [4] and [6].

Equations (6) and (7), together with the equation for the coupling strength [Eq. (9)], fully describe the polarization behavior of a VCSEL. In Sec. IV we will discuss the influence of the inclusion of the carrier momentum on the polarization properties of the laser, and relate the microscopic description presented in this section to the macroscopic description given in Sec. II.

\section{COMBINING THE MACROSCOPIC AND MICROSCOPIC ANALYSIS}

In order to work toward a set of macroscopic rate equations, we divide the total population into reservoirs. Each reservoir contains a part of the population inversion that couples with a specific polarization, in our case circular polarization, linear polarization along [001], and linear polarization along [011]. Rate equations describe the population transfer between the various reservoirs, and the coupling between the reservoirs and the optical field.

The first step is to replace the interband transition by a distribution of transitions between discrete energy levels, 

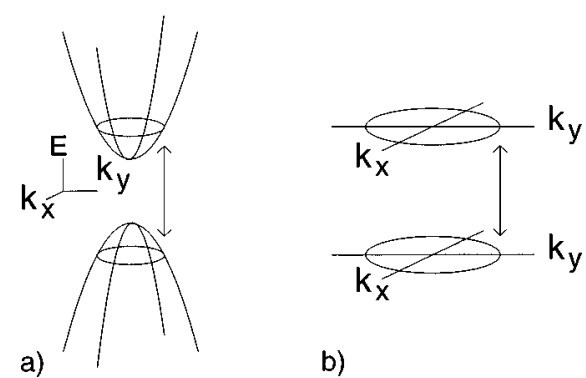

b)

FIG. 3. Illustration of (a) the interband transition, and (b) the transition between discrete energy levels for a fixed value of the magnitude of $\vec{k}_{\|}$.

where the distribution is over the orientation of $\vec{k}_{\|}$. We thus neglect spectral hole burning but do allow for momentum hole burning. This is illustrated in Fig. 3, where on the left side the band structure with parabolic bands is shown, and on the right hand side the transition between the discrete energy levels. In order to make this approximation we replace the summation over the momentum states by an integration, using $\theta$ and $\phi$ as integration variables: $\Sigma_{\vec{k}}=\int d \theta \int d \phi \rho(\phi)$ Here we introduced $\rho(\phi)$ as the reduced density of states [17]. The density of states (DOS) is related to the effective masses of the conduction and valence band, which in principle depend on the orientation of $\vec{k}_{\|}$with respect to the crystalline axes. Because the orientational dependence will reflect the cubic symmetry of the system, we introduce, for the dependence of the DOS on the orientation, $\rho(\phi)=(\bar{\rho}$ $+\Delta \rho \cos 4 \phi) / 2 \pi$. Because the bands are taken to be parabolic, the DOS does not depend on $\theta$. After replacing the summation by an integral we integrate out the $\theta$ dependence and introduce an average value for $\theta$, indicated by $\bar{\theta}$. In doing this we neglect the influence of the magnitude of $\vec{k}_{\|}$, and focus on the influence of the orientation of the carrier momentum.

As a second step we introduce carrier reservoirs, i.e., subcategories of the carriers. By combining Eqs. (6) and (9) we find, for the optical field amplitude,

$$
\begin{aligned}
\dot{E}= & -\kappa E+2 \kappa(1-i \alpha)\left[g_{0} \bar{N}+\left(g_{1} \Delta N_{1}\right) S_{1}+\left(g_{1} \Delta N_{2}\right) S_{2}\right. \\
& \left.+\left(g_{3} \Delta N_{3}\right) S_{3}\right] E .
\end{aligned}
$$

Here $g_{i}$ are the coefficients in the coupling strength [Eq. (9)] for the average value of $\theta: g_{0}=\left(\frac{1}{4}\right)\left(1+\cos ^{2} \bar{\theta}\right), \quad g_{1}=$ $-\left(\frac{1}{4}\right) \sin ^{2} \bar{\theta}$, and $g_{3}=\left(\frac{1}{2}\right) \cos \bar{\theta}$. Furthermore we defined the population reservoirs that couple with specific polarizations:

$$
\begin{gathered}
\Delta N_{1}=\frac{1}{2} \int d \phi \rho(\phi)\left(N_{+}(\phi)+N_{-}(\phi)\right) \cos 2 \phi \\
\Delta N_{2}=\frac{1}{2} \int d \phi \rho(\phi)\left(N_{+}(\phi)+N_{-}(\phi)\right) \sin 2 \phi \\
\Delta N_{3}=\frac{1}{2} \int d \phi \rho(\phi)\left(N_{+}(\phi)-N_{-}(\phi)\right), \\
\bar{N}=\frac{1}{2} \int d \phi \rho(\phi)\left(N_{+}(\phi)+N_{-}(\phi)\right) .
\end{gathered}
$$

Here $N_{s}(\phi)=N_{s, \vec{k}}$, with the orientation of $\vec{k}$ determined by the angles $\bar{\theta}$ and $\phi$.

In Eq. (13) the polarization dependence of the linear gain has been included through the Stokes parameters $S_{1,2,3}$. The real part of the prefactors of $S_{1,2,3}$ determine the gain or loss difference between the polarizations that correspond to $S_{1,2,3}=+1$ and $S_{1,2,3}=-1$, while the imaginary parts of these prefactors determine the corresponding birefringence, which is a factor $\alpha$ larger for all terms. For instance, if $\Delta N_{1}$ is nonzero there will be a gain or loss difference and a birefringence between light polarized along [001] (for which $S_{1}=1$ and $S_{2}=S_{3}=0$ ) and light polarized along [010] (for which $S_{1}=-1$ and $\left.S_{2}=S_{3}=0\right)$. In the same way $\Delta N_{2}$ is related to an anisotropy between light polarized along [011] and $[01 \overline{1}]$, and $\Delta N_{3}$ is related to an anisotropy between leftand right-handed circularly polarized light. The polarization independent part of the gain is determined by $\bar{N}$.

As a third step we write the rate equations that describe the population transfer between the various reservoirs:

$$
\begin{aligned}
\dot{\bar{N}}= & -\gamma(\bar{N}-\bar{R})-2 \gamma\left(g_{0} \bar{N}+g_{1} S_{1} \Delta N_{1}+g_{1} S_{2} \Delta N_{2}\right. \\
& \left.+g_{3} S_{3} \Delta N_{3}\right) I, \\
\Delta \dot{N}_{1}= & -\gamma\left(\Delta N_{1}-\Delta R_{1}\right) \\
& -2 \gamma\left(g_{0} \Delta N_{1}+\frac{1}{2} g_{1} S_{1}\left\{1+\frac{\Delta \rho}{2 \bar{\rho}}\right\} \bar{N}\right) I-\gamma_{k} \Delta N_{1},
\end{aligned}
$$

$$
\begin{aligned}
\Delta \dot{N}_{2}= & -\gamma\left(\Delta N_{2}-\Delta R_{2}\right) \\
& -2 \gamma\left(g_{0} \Delta N_{2}+\frac{1}{2} g_{1} S_{2}\left\{1-\frac{\Delta \rho}{2 \bar{\rho}}\right\} \bar{N}\right) I-\gamma_{k} \Delta N_{2},
\end{aligned}
$$

$\Delta \dot{N}_{3}=-\gamma\left(\Delta N_{3}-\Delta R_{3}\right)-2 \gamma\left(g_{0} \Delta N_{3}+g_{3} S_{3} \bar{N}\right) I-2 \gamma_{j} \Delta N_{3}$

Here the various components of the normalized pump rate $\left(\bar{R}\right.$ and $\left.\Delta R_{i}\right)$ are defined in the same way as the population reservoirs in Eqs. (14)-(17), including the factors $\frac{1}{2}$. In the remaining part of this section we will use Eqs. (13) and (18)-(21) to describe the linear and nonlinear polarization properties of the QW gain medium.

First we look at linear effects, i.e., we neglect the saturation terms $\left(\bar{R}-1 \ll 1,|E|^{2} \ll 1\right)$, but we take into account the fact that the pumping can be anisotropic. The steady-state populations are then

$$
\begin{gathered}
\bar{N}=1, \\
\Delta N_{1}=\frac{\Delta R_{1}}{\Gamma_{k}}, \\
\Delta N_{2}=\frac{\Delta R_{2}}{\Gamma_{k}},
\end{gathered}
$$




$$
\Delta N_{3}=\frac{\Delta R_{3}}{\Gamma_{s}}
$$

Here we introduced.

$$
\begin{gathered}
\Gamma_{s}=1+2 \gamma_{j} / \gamma, \\
\Gamma_{k}=1+\gamma_{k} / \gamma .
\end{gathered}
$$

So the anisotropies in the population distribution are determined by the balance between the anisotropies in the pump and the relaxation rates. Using Eq. (13), this leads to the following expression for the optical amplitude:

$$
\begin{aligned}
\frac{d}{d t} E= & -\kappa E+2 \kappa(1-i \alpha)\left[g_{0}+\left(\frac{g_{1}}{\Gamma_{k}} \Delta R_{1}\right) S_{1}+\left(\frac{g_{1}}{\Gamma_{k}} \Delta R_{2}\right) S_{2}\right. \\
& \left.+\left(\frac{g_{3}}{\Gamma_{s}} \Delta R_{3}\right) S_{3}\right] E
\end{aligned}
$$

It can be seen that the anisotropic pumping creates dichroism and birefringence. In optically pumped VCSEL's the anisotropic pump originates from the polarization of the pump beam. Circularly polarized pump light creates a difference in pump rates between the spin levels, which means that $\Delta R_{3}$ is nonzero. Linearly polarized pump light creates a difference in pump rates between the carrier states with the momentum parallel to the optical polarization of the pump, and states with the momentum perpendicular, which causes $\Delta R_{1}$ and/or $\Delta R_{2}$ to be nonzero. The magnitudes of the anisotropies that are created by this polarized pumping depend on the relaxation parameters of the populations: $\Gamma_{s}$ for circular polarizations and $\Gamma_{k}$ for linear polarizations.

Next we study the nonlinear anisotropies in the presence of an isotropic pump $\left(\Delta R_{1,2,3}=0\right)$. This is the case that naturally occurs in electrically pumped VCSEL's. We adiabatically eliminate the inversion in order to compare our results to the macroscopic description from Sec. II. We again look at the steady-state populations, neglecting terms of order higher than $|E|^{2}$ :

$$
\begin{gathered}
\bar{N} \approx \bar{R}\left(1-2 g_{0} I\right), \\
\Delta N_{1}=-\frac{g_{1} S_{1}}{\Gamma_{k}}\left\{1+\frac{\Delta \rho}{2 \bar{\rho}}\right\} \bar{N} I, \\
\Delta N_{2}=-\frac{g_{1} S_{2}}{\Gamma_{k}}\left\{1-\frac{\Delta \rho}{2 \bar{\rho}}\right\} \bar{N} I, \\
\Delta N_{3}=-2 \frac{g_{3} S_{3}}{\Gamma_{s}} \bar{N} I .
\end{gathered}
$$

If we use the steady-state populations to calculate the polarization evolution, we find

$$
\begin{aligned}
\frac{d}{d t} E= & -\kappa E+2 \kappa(1-i \alpha) g_{0} \bar{R} E-4 \kappa(1-i \alpha) \bar{R} \\
& \times\left[g_{0}^{2}+\frac{\left(g_{1} S_{1}\right)^{2}}{2 \Gamma_{k}}\left(1+\frac{\Delta \rho}{2 \bar{\rho}}\right)+\frac{\left(g_{1} S_{2}\right)^{2}}{2 \Gamma_{k}}\left(1-\frac{\Delta \rho}{2 \bar{\rho}}\right)\right. \\
& \left.+\frac{\left(g_{3} S_{3}\right)^{2}}{\Gamma_{s}}\right] I E .
\end{aligned}
$$

From this equation we want to deduce the values for the parameters $\delta_{r, i}$ and $\sigma_{r, i}$. The nonlinear refractive index and nonlinear gain coefficients for a specific eigenpolarization can be determined from Eq. (33) by using the corresponding set of Stokes parameters and comparing to Eq. (5): $S_{1}, S_{2}, S_{3}=1,0,0$ yields $n_{2}^{(0)}$ and $\beta^{(0)} ; S_{1}, S_{2}, S_{3}=0,1,0$ yields $n_{2}^{(45)}$; and $\beta^{(45)}$ and $S_{1}, S_{2}, S_{3}=0,0,1$ yields $n_{2}^{\text {(circ) }}$ and $\beta^{\text {(circ) }}$. In this way we find, using Eqs. (1)-(4),

$$
\begin{gathered}
\delta_{i}=1-\frac{g_{0}^{2}+g_{3}^{2} / \Gamma_{s}}{g_{0}^{2}+\frac{g_{1}^{2}}{2 \Gamma_{k}}\left(1+\frac{\Delta \rho}{2 \bar{\rho}}\right)} \\
\approx-\frac{g_{3}^{2}}{g_{0}^{2}} \frac{1}{\Gamma_{s}}+\frac{g_{1}^{2}}{g_{0}^{2}} \frac{1}{2 \Gamma_{k}}\left(1+\frac{\Delta \rho}{2 \bar{\rho}}\right), \\
\frac{\delta_{r}=\delta_{i},}{g_{0}^{2}+\frac{g_{1}^{2}}{2 \Gamma_{k}}\left(1-\frac{\Delta \rho}{2 \bar{\rho}}\right)} \\
g_{0}^{2}+\frac{\Delta \rho}{2 \Gamma_{k}^{2}}\left(1+\frac{\Delta \rho}{2 \bar{\rho}}\right)
\end{gathered}
$$

Thus we have fully characterized the polarization dependence of the saturation. The expressions for $\delta_{r, i}$ and $\sigma_{r, i}$ deviate from the expressions found in the SFM model. The parameter $\delta_{r, i}$, which is equal to $-1 / \Gamma_{s}$ in the SFM model, now depends on the relative orientation of the carrier momentum (via the coefficients $g_{0,1,3}$ ), on the momentum relaxation $\left(\operatorname{via} \Gamma_{k}\right.$ ), and on the anisotropy in the DOS (via $\Delta \rho / \bar{\rho}$ ). Relaxation effects associated with the carrier spin cause $\delta_{r, i}$ to be negative [first term in Eqs. (35) and (34)], while relaxation effects associated with the momentum make a positive contribution to $\delta_{r, i}$ [second term in Eqs. (35) and (34)]. So the reservoir based on the spin, $\Delta N_{3}$, creates a preference for the emission of linearly polarized light, while the reservoirs based on the carrier momentum, $\Delta N_{1}$ and $\Delta N_{2}$, create a preference for circularly polarized light. Note that even if the momentum decay occurs very rapidly (a very large $\Gamma_{k}$ ) there is still a correction to the value of $\delta_{r, i}$ as compared to the SFM model. Only if the in-plane component of the carrier momentum is zero will the expression for $\delta_{r, i}$ reduce to that found from the SFM model. The anisotropy parameter $\sigma_{r, i}$, which is zero in the SFM model, is nonzero if the DOS is anisotropic $(\Delta \rho \neq 0)$. However, because $\sigma_{r, i}$ is inversely proportional to $\Gamma_{k}$, a large momentum decay rate will reduce the effect of an anisotropic DOS. 


\section{DISCUSSION}

In this section we will give a qualitative estimate of the importance of the various effects discussed in this paper, by looking at a "typical" QW, i.e., 10-nm GaAs in an $\mathrm{Al}_{0.2} \mathrm{Ga}_{0.8} \mathrm{As}$ cladding. The physical parameters that play a role are the in-plane component of the carrier momentum, the relaxation parameters for the carrier momentum $\Gamma_{k}$ and carrier spin $\Gamma_{s}$, and the anisotropy in the DOS $\Delta \rho / \bar{\rho}$.

The orientation of the carrier momentum is determined by the relative magnitudes of $k_{\perp}$ and $k_{\|}$, where $k_{\perp}$ is the component of the carrier momentum perpendicular to the $\mathrm{QW}$, which can be obtained from QW thickness and the $\mathrm{GaAs} / \mathrm{Al}_{0.2} \mathrm{Ga}_{0.8} \mathrm{As}$ material parameters [18]. Although it is difficult to give an exact value for the magnitude of $k_{\|}$, as a typical value we use $k_{\|}=0.2 \mathrm{~nm}^{-1}$ [19]. This value is determined by (i) the filling of the states in the conduction and valence bands, (ii) the thermal (Fermi-Dirac) distribution in these bands, and (iii) intraband relaxation effects. In order to determine the coupling strength we need the average angle between the direction perpendicular to the QW and the carrier momentum, which was called $\bar{\theta}$ in Sec. IV. For $k_{\|}$ $=0.2 \mathrm{~nm}^{-1}$ and $k_{\perp}=0.25 \mathrm{~nm}^{-1}$, we find $\bar{\theta}=39^{\circ}$.

Next we address the relaxation parameters for the carrier spin $\Gamma_{s}$ and the carrier momentum $\Gamma_{k}$. In Ref. [4], $\Gamma_{s}$ was estimated on the basis of literature data to be between 1 and 200, using the definition as in Eq. (26). The uncertainty in this estimate lies in the spin-flip rate $\gamma_{j}$, which depends strongly on carrier density, temperature, and QW geometry. A first experimental estimate was obtained from studying the polarization behavior of VCSEL's in an axial magnetic field, which yielded $75<\Gamma_{s}<150$ [20]. More recently $\Gamma_{s}$ was determined by using optically pumped VCSEL's and studying the influence of the ellipticity of the pump polarization on the VCSEL polarization. By measuring the pump-induced dichroism and birefringence, as described in Eq. (28), $\Gamma_{s}$ was estimated to be between 150 and 450 [8]. Here the main uncertainty stems from the cavity loss rate, which is a parameter that is very difficult to determine experimentally. The most recent experimental estimate for $\Gamma_{s}$ was from a direct measurements of the polarization dependence of the saturation, which yielded estimates for $\Gamma_{s}$ between 100 and 500 [7]. Again the main uncertainty stems from the cavity loss rate. The conclusion is therefore that from independent measurements in practical VCSEL's $\Gamma_{s}$ is estimated to be between 200 and 400 .

Using a measurement of the momentum relaxation rate, $\tau=0.1 \mathrm{ps}$ [9], $\Gamma_{k}$ can be estimated to be between $10^{3}$ and $10^{4}$. As in the spin case $\Gamma_{k}$ can also be estimated from the influence of the polarization of the pump in optically pumped VCSEL's. For $\Gamma_{k}$ it is the orientation of the pump polarization that plays a role, as compared to the ellipticity for $\Gamma_{s}$. In Ref. [8] it was observed that the influence of the pump polarization orientation was about 20 times as small as the influence of the pump ellipticity. From Eq. (28) it can be seen that this corresponds to $\Gamma_{k}$ being about 20 times as large as $\Gamma_{s}$. We thus find $\Gamma_{k}$ to be between $4 \times 10^{3}$ and $8 \times 10^{3}$.

The optical properties of the QW are determined by the reduced DOS [17], in which both the conduction-band DOS and the valence-band DOS are incorporated. Using the material parameters as in, for instance, Refs. [21] and [18], we estimate the anisotropy in the reduced DOS, $\Delta \rho / \bar{\rho}$, to be approximately $3 \%$.

Now we are in a position to examine the parameters that determine the polarization dependence of the saturation. In Eqs. (34) and (35) the expression for $\delta_{r, i}$ contains two terms. The first term depends on $\Gamma_{s}$ and on $\bar{\theta}$ (via $g_{3}$ and $g_{1}$ ), while the second term is dominated by $\Gamma_{k}$. Because of the large value of $\Gamma_{k}$ and because $g_{1}^{2}<g_{3}^{2}$ the contribution of the second term is small, so the value for $\delta_{r, i}$ is largely determined by the magnitude of the in-plane carrier momentum and the spin relaxation. We find $\delta_{r, i}=-0.94 / \Gamma_{s} \approx 3 \times 10^{-3}$. In spite of the large in-plane component of the carrier momentum, the correction to $\delta_{r, i}$, as compared to the SFM model, is only $6 \%$. Due to the large uncertainty in $\Gamma_{s}$ this discrepancy cannot be observed experimentally.

In theory there should also be a cubic term; after substitution of the parameters estimated above the anisotropy parameter $\sigma_{r, i}$ [see Eqs. (36) and (37)] is found to have a small value, $\sigma_{r, i}=3 \times 10^{-7}$. So the dependence of the saturation intensity on the polarization orientation is four orders of magnitude smaller than the dependence on the polarization ellipticity. This small value is due to the large value of the momentum decay rate, and the limited anisotropy in the reduced DOS.

At the end of this section we want to address qualitatively an aspect that has been ignored until now namely, the bandmixing between the $\mathrm{HH}$ and $\mathrm{LH}$ valence bands. Band mixing occurs for $k_{\|}>0$, and is stronger if the HH and LH bands are close. Due to band mixing the interband transition dipole element changes. The magnetic sublevels are now coupled by elliptically, instead of circularly, polarized light, where the orientation of the polarization ellipse depends on orientation of the carrier momentum. So linearly polarized light will couple preferentially with carriers with $\vec{k}_{\|}$either parallel or perpendicular to the optical polarization. Whether it is parallel or perpendicular depends on the detailed band structure. Thus the effects of a nonideal QW as described in this paper are enhanced by band mixing. It might be interesting to explore whether it is possible, by means of band-structure engineering, to create a band structure such that it is linearly polarized light that couples the magnetic sublevels. In this case the sign of the parameter $\delta$ would change from negative to positive, i.e., the intrinsic (nonlinear) preference of the QW for linear polarization would change to a preference for circularly polarized light. There are some complications to this idea. In order to obtain strong band mixing, the $\mathrm{HH}$ and LH bands have to be close. So the fact that there are two valence bands at different energies has to be included in the model. Whether band mixing can play a significant role in the polarization properties of practical VCSEL's remains to be seen.

\section{CONCLUSION}

In this paper we have presented an extension of the SFM model for the polarization of QW VCSEL's, taking into account the fact that the component of the carrier momentum in the plane of the QW is roughly as large as the transverse component. First we have shown from macroscopic symmetry arguments that a limited number of parameters $\delta_{r, i}$ and 
$\sigma_{r, i}$ characterize the polarization-dependent saturation in a cubic crystal. All these parameters depend on the magnitude of the in-plane component of the carrier momentum. The parameters $\delta_{r, i}$, which describe the dependence of the saturation on the ellipticity, are dominated by the spin-flip rate $\Gamma_{s}$, and to a lesser extent by the momentum relaxation rate $\Gamma_{k}$. The parameters $\sigma_{r, i}$, which describe the influence of the cubic symmetry of GaAs, depend mainly on $\Gamma_{k}$ and on the anisotropy in the DOS. The polarization dependence of the saturation is manifested in a (polarization-dependent) nonlinear refractive index, parametrized by $\delta_{r}$ and $\sigma_{r}$, and a nonlinear absorption coefficient, parametrized by $\delta_{i}$ and $\sigma_{i}$.

For a typical QW (10-nm GaAs) the polarization dependence of the nonlinear refractive index and absorption coefficient are equally large. The correction to $\delta_{r, i}$ was about $6 \%$ as compared to the SFM model. At present this is within the experimental uncertainty, which is limited by the uncertainty in $\Gamma_{s}$. The anisotropy parameters $\sigma_{r, i}$ were found to be nonzero, but still very small in our model. The dependence of the saturation on the orientation of linearly polarized light due to the cubic crystalline symmetry is about four orders of magnitude smaller than the dependence on the ellipticity.

Furthermore we studied the effect of a nonequilibrium carrier distribution in an isotropic crystal. A nonuniform spin distribution creates circular dichroism and birefringence, as in the SFM model. We have shown that likewise an anisotropic momentum distribution creates linear birefringence and dichroism. This latter effect is absent in the SFM model. The experimental observation of the influence of a nonequilibrium momentum distribution was used in Ref. [8] to estimate the momentum decay parameter in a practical QW.

From our extended theory it is clear why the SFM model, which neglects $\vec{k}_{\|}$, works relatively well. With respect to the polarization-dependent saturation we have shown that the role of the carrier momentum distribution is much smaller than the role of the carrier spin distribution. This is due to the fact that the relaxation rate of a nonequilibrium momentum distribution is much larger than the relaxation rate for the carrier spin: effectively the momentum distribution is always in equilibrium. Nonlinear anisotropies related to the carrier momentum are therefore much smaller than anisotropies related to the carrier spin.

\section{ACKNOWLEDGMENTS}

This work is part of the research program of the "Stichting voor Fundamenteel Onderzoek der Materie (FOM)." We acknowledge support from the European Union under the ESPRIT Project No. 20029, ACQUIRE, and TMR Network No. ERB4061 PL951021.
[1] A. K. Jansen van Doorn, M. P. van Exter, and J. P. Woerdman, Appl. Phys. Lett. 69, 1041 (1996).

[2] R. F. M. Hendriks, M. P. van Exter, J. P. Woerdman, A. van Geelen, L. Weegels, K. H. Gulden, and M. Moser, Appl. Phys. Lett. 71, 2599 (1997).

[3] A. K. Jansen van Doorn, M. P. van Exter, A. M. van der Lee, and J. P. Woerdman, Phys. Rev. A 55, 1473 (1997).

[4] M. San Miguel, Q. Feng, and J. V. Moloney, Phys. Rev. A 52, 1728 (1995).

[5] H. F. Hofmann and O. Hess, Phys. Rev. A 56, 868 (1997).

[6] M. P. van Exter, R. F. M. Hendriks, and J. P. Woerdman, Phys. Rev. A 57, 2080 (1998).

[7] M. P. van Exter, A. Al-Remawi, and J. P. Woerdman, Phys. Rev. Lett. 80, 4875 (1998).

[8] R. F. M. Hendriks, M. P. van Exter, and J. P. Woerdman, IEEE J. Quantum Electron. 34, 1455 (1998).

[9] J. L. Oudar, A. Migus, D. Hulin, G. Grillon, J. Etchepare, and A. Antonetti, Phys. Rev. Lett. 53, 384 (1984).

[10] T. D. Krauss, J. K. Ranka, F. W. Wise, and A. L. Gaeta, Opt. Lett. 20, 1110 (1995).

[11] D. C. Hutchings, Opt. Lett. 20, 1607 (1995).

[12] W. A. Schroeder, D. S. McCallum, D. R. Harken, M. D.
Dvorak, D. R. Andersen, and A. L. Smirl, J. Opt. Soc. Am. B 12, 401 (1995).

[13] M. Sargent, M. O. Scully, and W. E. Lamb, Laser Physics (Addison-Wesley, Reading, MA, 1974).

[14] H. Shen, M. Wraback, J. Pamulapati, P. G. Newman, M. Dutta, Y. Lu, and H. C. Kuo, Phys. Rev. B 47, 13933 (1993).

[15] M. P. van Exter, A. K. Jansen van Doorn, and J. P. Woerdman, Phys. Rev. A 56, 845 (1997).

[16] Y-.G. Ju, Y-.H. Lee, H-.K. Shin, and I. Kim, Appl. Phys. Lett. 71, 741 (1997).

[17] S. W. Corzine, R.-H. Yan, and L. A. Coldren, in Quantum Well Lasers, edited by P. S. Zory (Academic, New York, 1993).

[18] W. W. Chow, S. W. Koch, and M. S. Sargent III, Semiconductor-Laser Physics (Springer-Verlag, Berlin, 1994).

[19] F. Jahnke, K. Henneberger, W. Schäfer, and S. W. Koch, J. Opt. Soc. Am. B 10, 2394 (1993).

[20] A. K. Jansen van Doorn, M. P. van Exter, M. Travagnin, and J. P. Woerdman, Opt. Commun. 133, 252 (1997).

[21] R. P. O'Reilly and Alli Ghiti, in Quantum Well Lasers, (Ref. [17]), Chap. 7. 